\title{
FACTORES DE RIESGO E INCIDENCIA DE INFECCIÓN DEL SITIO QUIRÚRGICO. HOSPITAL DEL INSTITUTO ECUATORIANO DE SEGURIDAD SOCIAL DE BABAHOYO, ECUADOR, 2015
}

\author{
INCIDENCE AND RISK FACTORS OF SURGICAL SITE INFECTION. ECUADORIAN SOCIAL \\ SECURITY HOSPITAL, BABAHOYO, 2015
}

FATORES DE RISCO E INCIDÊNCIA DE INFECÇÃO DO LOCAL CIRÚRGICO. HOSPITAL DO INSTITUTO EQUATORIANO DE SEGURANÇA SOCIAL DE BABAHOYO-EQUADOR. 2015

\author{
HERMAN ROMERO RAMÍREZ1', MARITZA PLACENCIA MEDINA² \\ ${ }^{1}$ Universidad Técnica de Babahoyo, Babahoyo, Ecuador \\ 2 Universidad Nacional Mayor de San Marcos, Lima, Perú
}

\begin{abstract}
Resumen Introducción: la infección del sitio quirúrgico (ISQ) es la complicación posoperatoria más frecuente en el ámbito hospitalario. En su etiopatogenia contribuyen factores de tipo intrínseco y extrínseco, cuya identificación nos orienta a tomar medidas preventivas para disminuir las tasas de morbilidad y mortalidad de esta complicación. Objetivo: determinar la relación entre factores de riesgo e incidencia de infección del sitio quirúrgico. Materiales y métodos: estudio observacional, descriptivo y enfoque cuantitativo, se calcularon medidas de frecuencias (absolutas y relativas) y mediante modelo de regresión logística para el análisis bivariado y multivariado. Se determinaron los casos con ISQ utilizándose el análisis bivariado con prueba de Chi cuadrado; y, multivariado, con regresión logística (odds ratio) para la asociación entre los factores de riesgo e ISQ. Resultados: la edad promedio de pacientes con ISQ fue de 54 años. Se presentó infección en el $20 \%$ de los pacientes que tuvieron desnutrición y neoplasias. El tiempo quirúrgico mayor a 3 horas $(O R=4,502)$ y el uso de drenes $(O R=1,560)$ se asociaron con ISQ. La tasa de incidencia de ISQ fue de 4,1\%. Conclusiones: los factores de riesgo que más se relacionaron con ISQ fueron: edad, tiempo quirúrgico, uso de drenes, neoplasias y desnutrición.

PALABRAS CLAVE: factores de riesgo, infección del sitio quirúrgico, incidencia.
\end{abstract}

Abstract

Introduction. surgical site infection (SSI) is the most frequent postoperative complication in a hospital setting. Pathogenic factors of SSI are both intrinsic and extrinsic and their recognition allows medical professionals to take preventive measures to reduce mortality and morbidity rates. Objetive: To determine the association between risk factors and SSI incidence. Methods. this is an observational, quantitative and descriptive study. Frequency measures (relative and absolutes) were calculated, bivariate analysis with chi square was used to identify the SSI cases and a multivariate analysis with logistic regression (odds ratio) for the association between the variables. Results. the mean age for SSI patients was 54 years old. Infections were detected in $20 \%$ of patients with neoplasia and malnutrition. Surgical time was above 3 hours $(O R=4,502)$ and drainage devices use was associated with $\mathrm{SSI}(\mathrm{OR}=1,560)$. Incidence rate of SSI was 4,1\%. Conclusion. age, surgical site and time, drainage use, neoplasia and malnutrition were the risk factors more associated with SSI..

KEYWORDS: risk factors, incidence, infection.

Resumo Introdução: a infecção do sítio cirúrgico (ISC) é a complicação pós-operatória mais frequente no ambiente hospitalar. Em sua etiopatogenia contribuem fatores de tipo intrínseco e extrínseco, cuja identificação nos orientaria a tomar medidas preventivas para reduzir as taxas de morbimortalidade dessa infecção. Objetivo: determinar a relação entre fatores de risco e incidência de infecção do sítio cirúrgico. Materiais e métodos: estudo observacional descritivo e abordagem quantitativa. Medições de frequência foram calculadas (absolutas e relativas) e por modelo de regressão logística para análise bivariada e multivariada. Os casos com ISC foram determinados utilizando análise bivariada com teste do qui-quadrado e multivariada com regressão logística (odds ratio) para a associação entre fatores de risco e ISC. Resultados: a idade média dos pacientes com ISC foi de 54 anos. A infecção ocorreu em $20 \%$ dos pacientes com desnutrição e neoplasias. Tempo cirúrgico superior a 3 horas $(O R=4,502)$ e 0 uso de drenos $(O R=1,560)$ foram associados à ISC. A taxa de incidência de ISC foi de 4,1\%. Conclusões: os fatores de risco mais relacionados à ISC foram idade, tempo cirúrgico, uso de drenos, neoplasias e desnutrição

PALAVRAS-CHAVE: fatores de risco, incidência, infecção. 
INTRODUCCIÓN

A mediados del siglo XIX, las infecciones en los pacientes operados constituían un 80 a 90 $\%$ de los casos. Después debido a algunas medidas de asepsia y antisepsia preconizadas por Semmelweis en 1851 y Lister en 1867, se redujo hasta $35 \%$ dichas infecciones. ${ }^{1}$ Posteriormente la aparición del autoclave de vapor (Koch-1880), el uso de bata y gorro en las salas de operaciones, así como la introducción de los guantes de goma estériles por Halsted 10 años después, contribuyeron a disminuir la tasa global de infecciones hasta menos del $10 \%$, y luego, con la utilización de los antibióticos, se logra una reducción aún mayor. $^{2}$

Para evitar las infecciones se dice que todas las cosas que tengan contacto con la herida quirúrgica deben estar estériles, sin la presencia de microorganismos. La piel intacta evitaría la infección por su acción protectora de barrera; por lo tanto la pérdida de su integridad la haría susceptible a la infección, ya que se crea una puerta de entrada de microorganismos; cualquier elemento contaminado que entre en contacto con la piel, se considera riesgo de infección. ${ }^{3}$

Como factor de riesgo de infección del sitio quirúrgico (ISQ) se entiende toda variable que presenta una relación independiente y significativa con el desarrollo de una infección tras una intervención quirúrgica. Evitar todos los riesgos es poco probable porque algunos son inherentes al paciente o a las prácticas sanitarias; sin embargo muchos sí podrían modificarse. El conocimiento de los factores de riesgo y la adherencia a las medidas de prevención que gocen de buena evidencia científica, conducen a mejorar el proceso quirúrgico, a disminuir la tasa de infección y a elevar la calidad de la atención sanitaria del paciente. ${ }^{4}$

La ISQ representa la segunda causa más común de infecciones intrahospitalarias después de las infecciones de las vías urinarias, con consecuencias en los costos, estancia hospitalaria, incapacidades y secuelas. ${ }^{5}$

El Comité de Prevención y Control de Infecciones Hospitalarias del hospital Rafael Ángel Calderón, Guardia (2005), documentó un porcentaje global del 11.5\% de infecciones hospitalarias, de las cuales el 34\% correspondieron a infección de herida operatoria y el $28 \%$ a las vías respiratorias. ${ }^{6}$
A nivel mundial, la tasa de infección del sitio quirúrgico oscila entre el $2,5 \%$ a $41,9 \%$ en las heridas limpias contaminadas hasta las heridas sucias ${ }^{7}$ y se puede incrementar si existen factores de riesgo asociados. Estos factores que propician el desarrollo de ISQ pueden ser de 2 tipos: 1) factores propios del paciente (intrínsecos) y 2) factores relacionados a su entorno (extrínsecos).

También ha sido controversial la práctica de la eliminación del vello en el preoperatorio y su relación con la infección del sitio quirúrgico. En relación a ésto, un estudio basado en evidencias, The Joanna Briggs Institute demostró que la eliminación del vello del sitio operatorio no reduce el riesgo de infección. Sin embargo, se lo debe considerar para permitir un mejor acceso quirúrgico y tener un óptimo campo visual. ${ }^{8} \mathrm{La}$ fuente de infección para gran parte de las ISQ son los gérmenes de la flora endógena de la piel y mucosas del paciente, así como infecciones en otra parte de su organismo. ${ }^{9}$

En un estudio realizado en 1990, sobre el impacto de las ISQ, se encontró que el incremento de los costos hospitalarios en los pacientes que presentaban infecciones de herida quirúrgica era superior frente a los que no la tenían, como también la mortalidad se duplicó en estos casos, ya que esta complicación genera prolongación de la estancia hospitalaria. ${ }^{10}$ En otro estudio realizado en España en 1999 por Vaqué, para servicios quirúrgicos, el incremento de la estancia hospitalaria en pacientes infectados fue en promedio de 10,2 días, dependiente del número de infecciones pudiendo llegar hasta 25,2 días. ${ }^{11}$

Los pacientes que desarrollan una ISQ tienen un $60 \%$ más de probabilidad de ingresar en una unidad de cuidados intensivos, 5 veces más de reingresar al hospital y el doble de posibilidades de fallecer que los pacientes sin infección. ${ }^{12}$

El presente estudio se realizó para identificar características o factores de riesgo de infección del paciente: edad, sexo, comorbilidades, tiempo de duración de los procedimientos operatorios, especialidad quirúrgica y uso de drenes. El objetivo fue determinar los factores de riesgo relacionados con las ISQ en pacientes operados de cirugías programadas o electivas en el hospital del Instituto Ecuatoriano de Seguridad Social (IESS) de Babahoyo, Ecuador en el 2015. 


\section{MATERIALES Y MÉTODOS}

Se realizó un estudio observacional, y de diseño descriptivo; se consideraron los factores de riesgo propios del paciente (sexo, edad, comorbilidades) e inherentes al proceso quirúrgico (especialidad quirúrgica, tiempo empleado en la operación y uso de drenes); y la variable dependiente fue la infección del sitio quirúrgico, identificada por la presencia de dolor fuera de lo común en la herida, eritema y salida de secreción purulenta a través de la herida.

Se incluyeron a 219 pacientes intervenidos de cirugías programadas en el primer semestre del año 2015, seleccionados en forma aleatoria de una población de 512 y cumpliendo los siguientes criterios de inclusión: no tener infección evidente y pruebas de laboratorio analítica y clínica dentro de parámetros normales.

La información se recolectó mediante dos fichas (pre y postoperatorio) validadas previamente por expertos y se organizó la base de datos en el programa Excel 2007(Microsoft Corporation ${ }^{\circledR}$ ). Para el análisis estadístico se utilizó el programa SPSS versión 23 con la evaluación descriptiva de promedios y frecuencias de los factores de riesgo de ISQ; para el análisis comparativo de asociación entre los factores de riesgo e infección se utilizó el odds ratio (OR) modelo de regresión logístico, con un IC del $95 \%$ y para el análisis bivariado se utilizó la prueba estadística Chi cuadrado.

Las fuentes de información fueron: historias clínicas, notas de enfermería, observación directa del equipo médico y asistencial en las áreas de estudio. Durante el tiempo de la hospitalización se realizaron visitas al paciente diariamente, registrándose la evolución y la detección de presencia o ausencia de infección.

RESULTADOS

De los 219 pacientes, 109 (49,8\%) fueron varones y de éstos $5(4,5 \%)$ tuvieron ISQ; y $110(50,2 \%)$ mujeres, con $4(3,7 \%)$ casos de infección. La edad, osciló en el rango de 16 a 80 años, con un promedio de 39 años. Para el análisis bivariado, se la consideró como variable cuantitativa, y en el análisis multivariado, como posible factor de riesgo se la categorizó $(0: \leq 39$ años y $1: \geq$ 40 años).Se determinó el OR: 3,919; IC al 95\%: [0.730-21.049]. La edad promedio con y sin ISQ se visualiza en la figura 1 .

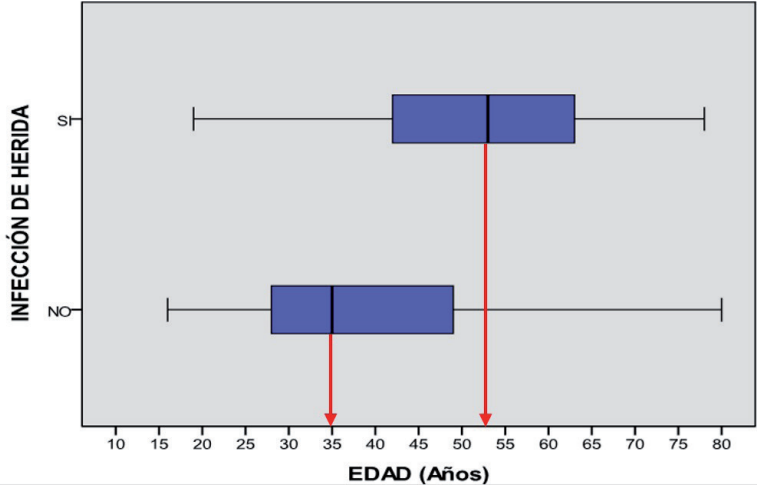

Figura 1. Relación de infección del sitio quirúrgico y promedio de edad

En la tabla1 se evidencian los datos referentes a las comorbilidades que presentaron los pacientes y su relación con ISQ.

TABLA 1. TABLA DE CONTINGENCIA ENTRE INFECCIÓN DE LA HERIDA Y COMORBILIDADES

\begin{tabular}{|c|c|c|c|}
\hline \multicolumn{4}{|c|}{ COMORBILIDADES, TABLA CRUZADA } \\
\hline \multicolumn{2}{|c|}{ INFECCIÓN DE SITIO QUIRÚRGICO } & \multicolumn{2}{|c|}{$\begin{array}{l}\text { RECUENTO DE \% } \\
\text { DENTRO DE INFECCIÓN DE HERIDE }\end{array}$} \\
\hline & & (NO) & (SI) \\
\hline \multirow[t]{2}{*}{ Diabetes mellitus } & No & $191 ; 95,9 \%$ & $19 ; 95 \%$ \\
\hline & $\mathrm{Si}$ & $8 ; 4,1 \%$ & $1 ; 5,0 \%$ \\
\hline \multirow{2}{*}{ Insuficiencia renal } & No & $197 ; 96,1 \%$ & $13 ; 92,8 \%$ \\
\hline & $\mathrm{Si}$ & $8 ; 3,9 \%$ & $1 ; 7,2 \%$ \\
\hline \multirow{2}{*}{ Neoplasias } & No & $206 ; 96,2 \%$ & $4 ; 80,0 \%$ \\
\hline & $\mathrm{Si}$ & $8 ; 3,8 \%$ & $1 ; 20,0 \%$ \\
\hline \multirow{2}{*}{ Cirrosis } & No & $206 ; 95,9 \%$ & $4 ; 100,0 \%$ \\
\hline & $\mathrm{Si}$ & $9 ; 4,1 \%$ & $0 ; 0,0 \%$ \\
\hline \multirow{2}{*}{ Desnutrición } & No & $206 ; 96,2 \%$ & $4 ; 80,0 \%$ \\
\hline & $\mathrm{Si}$ & $8 ; 3,8 \%$ & $1 ; 20,0 \%$ \\
\hline \multirow{2}{*}{ Obesidad } & No & $191 ; 95,5 \%$ & $19 ; 100,0 \%$ \\
\hline & $\mathrm{Si}$ & $9 ; 4,5 \%$ & $0 ; 0,0 \%$ \\
\hline
\end{tabular}

Con respecto a la especialidad quirúrgica el porcentaje más alto de ISQ lo tuvo traumatología con $10,25 \%$ (4 casos), seguido por urología con $4,65 \%$ ( 2 casos) y cirugía general 3,89 (3 casos). No se presentó ningún caso de infección en las intervenciones ginecológicas a pesar de haberse realizado un número importante de cirugías.

En relación al tiempo de duración de la cirugía, de 24 pacientes que fueron operados en un tiempo mayor a 3 horas, en $4(16,7 \%)$ de ellos se presentó ISQ; 124 fueron operados en un tiempo de 1 a 3 horas, de éstos $3(2,41 \%)$ tuvieron infección y de 71 procedimientos con duración menor a 1 hora, hubieron $2(2,9 \%)$ casos con infección del sitio quirúrgico. 
Para evitar inconvenientes de estimación en el modelo de regresión logística, se analizó la correlación entre las variables explicativas a través de la prueba de Chi-cuadrado con un nivel de significancia $\mathrm{p}<0.05$, con esta prueba se descartó la asociación entre estas variables explicativas, consideradas como posibles factores de riesgo de infección del sitio quirúrgico. En el análisis de los datos para relacionar la asociación entre la ISQ y los factores de riesgo, se observó que el OR mayor que 1 en la edad, indica que los pacientes con edad mayor a 40 años presentan un riesgo de infección en el sitio quirúrgico aproximadamente 4 veces más que los pacientes menores a 40 años (OR=3.919; IC 95\%: 0,730-21,0492).

Los pacientes con neoplasia presentaron aproximadamente 5 veces más riesgo de ISQ con relación a los pacientes que no tuvieron esta comorbilidad (OR=5.347; IC 95\%: 0,415-68,8842). La desnutrición fue considerada como un importante factor de riesgo de infección del sitio quirúrgico (OR=4.561; IC 95\%: 0,260-79,9292), indicándonos que los pacientes con desnutrición presentaron aproximadamente 5 veces más riesgo de tener infección en relación a los pacientes sin desnutrición. Se evidenció que el valor positivo de OR mayor a 1 en el tiempo quirúrgico, nos indicó que los pacientes que tuvieron cirugías con un tiempo mayor a 3 horas de duración, aumentó el riesgo de infección en el sitio quirúrgico aproximadamente 5 veces más en comparación con los pacientes que fueron intervenidos en menos de 1 hora ( $\mathrm{OR}=4.502$; IC 95\%: 0,319-32,7302), tabla 2 .

TABLA 2. ANÁLISIS MULTIVARIADO - REGRESIÓN LOGÍSTICA (ODDS RATIO): FACTORES DE RIESGO E ISQ

\begin{tabular}{|c|c|c|c|c|c|c|c|}
\hline & & 哭 & & & & $\begin{array}{l}\text { IC } 95 \% \\
\text { OR }\end{array}$ & PARA \\
\hline & $\boldsymbol{\infty}$ & 总悹 & 竧 & 山े & 뜸 & INFERIOR & SUPERIOR \\
\hline Edad (años) & 1,366 & 0,858 & 2,536 & 10,111 & 3,919 & 0,730 & 21,049 \\
\hline Neoplasias & 1,677 & 1,304 & 1,653 & 10,199 & 5,347 & 0,415 & 68,884 \\
\hline Desnutrición & 1,518 & 1,461 & 1,079 & 10,299 & 4,561 & 0,260 & 79,929 \\
\hline $\begin{array}{l}\text { Tiempo quirúr- } \\
\text { gico (< } 1 \text { hora) }\end{array}$ & & & 4,745 & 20,093 & & & \\
\hline $\begin{array}{l}\text { Tiempo } \\
\text { quirúrgico } \\
\text { (1 a } 3 \text { horas) }\end{array}$ & $\begin{array}{r}- \\
0,397\end{array}$ & 0,993 & 0,160 & 10,690 & 0,673 & 0,096 & 4,712 \\
\hline $\begin{array}{l}\text { Tiempo } \\
\text { quirúrgico } \\
>3 \text { horas } \\
\end{array}$ & 1,504 & 1,012 & 2,209 & 10,137 & 4,502 & 0,319 & 32,730 \\
\hline Uso de drenes & 0,445 & 0,803 & 0,306 & 10,580 & 1,560 & 0,323 & 7,528 \\
\hline Constante & 5,256 & 1,313 & 16,017 & 10,000 & 0,005 & & \\
\hline
\end{tabular}

Otra variable presente en el modelo final fue el uso de drenes, en este caso se evidenció que el riesgo de infección del sitio quirúrgico aumentó en aproximadamente $56 \%$ con relación al no uso de los mismos OR=1.5660 (IC 95\%: 1-1.560).

\section{DISCUSIÓN}

Las infecciones asociadas a la atención en salud son un evento frecuente a nivel hospitalario. Algunos factores relacionados con su aparición no son modificables (enfermedades concomitantes, gravedad del cuadro clínico y edad), pero el tipo de infección adquirida puede estar influenciado por el manejo que recibe el paciente durante la hospitalización. ${ }^{13}$

Según la literatura revisada, las infecciones de la herida quirúrgica más comunes después de una cirugía electiva son las producidas por estafilococos o enterobacterias. En la mayoría de los casos, la cura local soluciona el problema y, en ausencia de celulitis periincisional o sepsis sistémica, no son necesarios antibióticos por vía intravenosa y muchos resuelven de forma ambulatoria. ${ }^{14}$

Las ISQ siguen constituyendo un grupo importante dentro de las infecciones hospitalarias y en los países desarrollados representarían alrededor del $14 \%$.

Se han descrito muchos factores de riesgo relacionados a las ISQ; algunos dependientes del paciente, otros del proceso quirúrgico así como también del ambiente e infraestructura hospitalaria. ${ }^{15}$

A pesar de todos los avances científicos y tecnológicos en cirugía, las infecciones del sitio quirúrgico representan un porcentaje importante, siendo una causa de morbilidad a tener en cuenta.

En los años 70 y los 80 el riesgo de infección en cirugía se creía que era debido al tipo de la cirugía. En la actualidad se conoce que también depende de otros factores como los relacionados con el paciente, el procedimiento y el cirujano.

Con una adecuada evaluación preoperatoria se puede diagnosticar precozmente, alguna anomalía que haría cambiar los resultados del procedimiento quirúrgico a realizarse.

Para disminuir los posibles riesgos de una cirugía, se debe individualizar cada caso, tomando 
en cuenta las condiciones clínicas del paciente, así como otras características particulares. ${ }^{16}$

"La infección de la herida quirúrgica es un excelente indicador de calidad de las diferentes instituciones hospitalarias, y como tal, una prioridad para los departamentos de cirugía que buscan un manejo adecuado de esta entidad, en beneficio de los pacientes". ${ }^{17}$

La tasa de ISQ obtenida durante el período de estudio fue de $4.1 \%$, discretamente superior a las registradas por algunos servicios quirúrgicos y estudios internacionales, como la obtenida por Guanche Garcell, H, 2010 que fue de 3,8\% en igual tiempo y que aumentó hasta 4,8\% cuando se asoció a factores de riesgo. ${ }^{18}$

La Federación Latinoamericana de Cirugía, que establece valores de tasas de infección para las heridas limpias entre 1-5\%, también se encuentra en concordancia con el presente hallazgo hallazgo. Esta tasa de ISQ en cirugía limpia juega un importante papel como indicador de calidad de los servicios quirúrgicos. ${ }^{19}$

En otro estudio realizado por Daimilé López Tagle en el 2007 en Cuba, encontró una tasa global que oscila entre 2,6 y $2,9 \%$ por cada 100 egresos hospitalarios, con un promedio anual de 25 a 26 infectados; siendo antes las tasas hasta un $4,5 \%$, esta disminución quizás se deba al aumentar la rigidez en la aplicación de las normas de bioseguridad. ${ }^{20}$

Por otra parte se plantea que la infección del sitio quirúrgico es una de las principales complicaciones de las pacientes expuestas a cesárea, cuya incidencia oscila entre $1,46-30 \%$ de las operaciones, según el país de que se trate. ${ }^{21}$

Para predecir mejor el riesgode ISQ se han propuesto varios sistemas o programas (Por Ej. Study of Efficacy of Nosocomial Infection Control -SENIC y National Nosocomial Infection Surveillance Sistem - NNISS) de detección, control y seguimiento de heridas quirúrgicas que permiten a los médicos especialistas conocer los factores de riesgo, así como las complicaciones y las tasas de infección de las heridas quirúrgicas. Así mismo para valorar de mejor manera el riesgo de infección y de suministrar profilaxis o tratamiento antibiótico preoperatorio, es de gran utilidad la clasificación de las heridas quirúrgicas. ${ }^{8}$ Según la escala NNISS, el tiempo quirúrgico es el factor del índice NNIS que más influye en la tasa de infección (OR=3,43), ${ }^{5}$ esto estuvo en concordancia con nuestro estudio, en donde las intervenciones quirúrgicas desarrolladas en un tiempo mayora 3 horas de duración (odds ratio $\mathrm{OR}=4,502$ ) se consideró un factor de riesgo importante de ISQ.

Únicamente se pueden considerar como factor de riesgo las variables que tienen relación independiente y significativa con el desarrollo de una ISQ. La identificación de dichos factores permite una adecuada categorización de las distintas intervenciones quirúrgicas que se realizan, para así lograr un mejor control de las infecciones, tomando medidas preventivas como una adecuada asepsia y antisepsia, estabilización del estado clínico del paciente en lo relacionado a sus comorbilidades, profilaxis antimicrobiana, etc. ${ }^{22}$

A pesar de que es difícil modificar los factores dependientes del paciente, se podría minimizar algunos de estos efectos si se actúa con criterio preventivo para disminuir la incidencia de ISQ; en este estudio las neoplasias ( $\mathrm{OR}=5,347$; IC 95\%: 0,415-68,8842); $\mathrm{p}=0,199$ se consideraron como el principal factor de riesgo relacionado con infección, seguido de la desnutrición ( $\mathrm{OR}=4,561$; IC 95\%: 0,260 - 79,9292; $\mathrm{p}=0,299$ y de la edad (OR=3,919; IC 95\%: 0,730-21,0492; $\mathrm{p}=0,111$. En un trabajo de Katherine de la Rosa Muñoz "Riesgo de infección en pacientes post-quirúrgicos relacionado con las medidas de bioseguridad y el cuidado de heridas", se evidencia que el $67 \%$ tenían heridas contaminadas y solo un $33 \%$ tenían heridas limpias.

De los pacientes con heridas contaminadas, pertenecen a pacientes obesos en un $56 \%$ y por desnutrición $31 \%{ }^{19}$

Los factores relacionados con el entorno suelen ser de más fácil control que los factores de riesgo del paciente, ya que en su mayoría dependen de decisiones y acciones emprendidas por el personal asistencial del área de cirugía, así como también de la organización quirúrgica.

Entre los principales factores predisponentes del paciente, hay que tener presente también que el acto quirúrgico modifica la respuesta inmune además de factores como la edad avanzada, padecer de diabetes mellitus, cáncer, inmunodeficiencias, enfermedades cardiovasculares o respiratorias crónicas, desnutrición, alcoholismo, hipovitaminosis, etc. ${ }^{23}$ 
En relación con las comorbilidades los estudios coinciden en que la cardiopatía isquémica y la diabetes mellitus son las principales enfermedades que influyen en la ocurrencia de complicaciones de la herida quirúrgica en los pacientes operados. ${ }^{24}$

Algunos autores han destacado que la prolongación del acto quirúrgico eleva el riesgo de ISQ, especialmente cuando supera los 60 minutos, lo que se relaciona con nuestro estudio. De los factores analizados, dependientes del paciente destacan la edad, el sexo, las enfermedades asociadas, la especialidad quirúrgica, el tiempo operatorio y el uso de drenes. En cuanto a la edad y sexo nuestra serie no difiere significativamente de otras series, encontrando que el promedio de edad (54 años) en pacientes con ISQ fue mayor que en los que no presentaron infección de herida (35 años). De igual manera se estima que la incidencia de ISQ podría aumentar hasta 1.5 veces en pacientes mayores de 60 años, ${ }^{25}$ como lo revela en su estudio Ramos Luces et al, en 2011; así también lo mencionaron Alemán Mondeja y Guanche Garcell en 2001 en nuestra paciente con ISQ; reportan una incidencia mayor en pacientes mayores de 40 años. ${ }^{26}$

En la serie del presente estudio, las variables estudiadas al realizar un modelo de regresión logística, evidencian que la edad, neoplasias, desnutrición, el tiempo quirúrgico y el uso de drenes fueron variables predictora $(\mathrm{p}<0.05)$ de ISQ.

Las manifestaciones clínicas de la ISQ fueron comparables con las señaladas por otros autores. Gilbert encontró eritema de la zona en casi la totalidad de los pacientes, dolor en la mitad de los casos y secreción de pus en un tercio de ellos. ${ }^{27}$

En el estudio realizado por C. Arias, en Colombia,"la reducción en los costos en salud es una prioridad para cualquier institución. Uno de los objetivos de los programas de vigilancia es disminuir el costo de la hospitalización y el tratamiento de la infección”. Para reducir el riesgo de ISQ se debe tener presente que los riesgos pueden ser multifactoriales; están dados por las características del hospital, el equipo quirúrgico, el paciente y la cirugía. ${ }^{28}$

En su trabajo, Promis y Villablanca indicaron una tasa global de 3,4\% en 6826 operaciones limpias. Silva et al, en un análisis general de 4225 egresos quirúrgicos obtuvieron una tasa global de 2,3\%, ambas están por debajo de la encontrada en este estudio. ${ }^{25}$

La probabilidad de que una herida se infecte, depende en parte de que pueda contaminarse durante el acto quirúrgico. Para estimar las probabilidades de infección según el grado de contaminación, se han realizado algunos estudios como el de Altemeier, que creó un índice, mediante el cual pueden predecirse las posibilidades de infección del sitio quirúrgico. En base a esto se menciona que las cirugías clasificadas como contaminadas y sucias tienen un pronóstico desfavorable de infecciones posoperatorias. Según lo señalado anteriormente la tasa de infección del sitio quirúrgico de esta casuística fue de $3,2 \%$, inferior a la encontrada en el presente estudio que fue de 4,1\%; por Despaigne Alba et al, de 4,6\%, y por López Tagle et al en La Habana, de $10,9 \%{ }^{26}$

Con base en el presente estudio, la hipótesis de que los pacientes obesos tendrán más infecciones que los que registran menos peso, por la dificultad en el acceso al campo operatorio y prolongación del tiempo de cirugía; sin embargo, es probable que la desnutrición esté relacionada con infecciones del sitio quirúrgico, por carencias funcionales del sistema inmunológico y por la falta de nutrientes como la albúmina, necesaria en el proceso de cicatrización de las heridas. Los pacientes con bajo peso tuvieron más infecciones del sitio quirúrgico que los pacientes con peso normal o incluso sobrepeso; lo que determinó que un buen estado nutricional es necesario para evitar infecciones; este hallazgo también lo describieron Anderson y su equipo de trabajo. ${ }^{29}$

\section{CONCLUSIONES Y RECOMENDACIONES}

Se concluye que los factores de riesgo que más influyeron o se relacionaron con ISQ fueron la edad, desnutrición, neoplasias, tiempo quirúrgico mayor a 3 horas y uso de drenes. La edad promedio en los pacientes con ISQ fue de 54 años y la tasa de incidencia de infección en los pacientes operados fue de alrededor del $4,1 \%$. Una prevalencia de ISQ dentro del rango esperado en comparación a estudios previos en diversos hospitales a nivel nacional y mundial, sería necesario para seguir mejorando la adherencia de todo el personal asistencial de salud a las medidas destinadas a la prevención de este 
tipo de infección, mediante el entrenamiento continuo y supervisión constante por parte del comité de control y vigilancia de infecciones nosocomiales del hospital. ${ }^{30}$ Realizar estudios analíticos posteriores que permitan valorar el riesgo de infección de la herida quirúrgica, según factores de riesgo identificados.

\section{AGRADECIMIENTOS}

A los directivos del hospital del Seguro Social de Babahoyo-Ecuador por las facilidades brindadas para la realización de este estudio.

Al equipo de investigadores conformado por estudiantes de internado de la Escuela de Enfermería de la Facultad de Ciencias de la Salud de la Universidad Técnica de Babahoyo-Ecuador.

\section{REFERENCIAS BIBLIOGRÁFICAS}

1. Gálvez Vam. Factores personales e infecciosos asociados a la infección de herida quirúrgica, en el hospital militar central. [citado 27 de marzo de 2016]; Recuperado a partir de: http://www.inppares.org/revistasss/ Revista\%20IX\%202010/5-_Factores_Herida.pdf

2. De Atocha Castro-López M, Romero-Vázquez A. Factores de riesgo asociados a infección de heridas quirúrgicas en colecistectomía abierta electiva. Revisores que participaron en este número. 2010;16(1):869-74.

3. Nohora Isabel Tobo Vargas. Nursing staff and patient security vision in the operating room regarding asepsis and sterile technique. Av En Enferm. junio de 2013;31(1):159-69.

4. Ruiz SR, Pérez VL. Revista Metas de Enfermería: Factores de riesgo que contribuyen a la infección del sitio quirúrgico [Internet]. www.enfermeria21. com. Disponible en: http://www.enfermeria21.com/ revistas/metas/articulo/80942/

5. Londoño Á, Morales J, Murilla M. Características epidemiológicas y factores de riesgo relacionados con la infección en el sitio operatorio en procedimientos de cirugía general. Rev Chil Cir. 2011;63(6):559-65.

6. Guevara-Rodríguez M, Romero-Zúñiga JJ. Factores asociados a la infección hospitalaria de la herida operatoria en pacientes de cirugía limpia electiva en el Hospital Doutor Rafael Angel Calderón Guardia de Costa Rica. Acta Méd Costarric. 2010;52(3):159-66.

7. Castañeda Suárez ER. Factores de riesgo asociados a infección de sitio quirúrgico en pacientes adultos post operados de colecistectomía abierta. 2012 [citado 27 de marzo de 2016]; Recuperado a partir de: http://dspace.unitru.edu.pe:8080/xmlui/handle/123456789/196

8. Mendoza V, Dolores J, Celedón G, Hugo S, Morales V, Augusto C, et al. Prevalencia de infección del sitio quirúrgico en pacientes con cirugía abdominal. Cir Gen. marzo de 2011;33(1):32-7.

9. García HJ, Rodríguez-Medina X, Franco-Gutiérrez M, Miranda-Novales G, Villegas-Silva R. Factores de riesgo asociados a infección del sitio quirúrgico en recién nacidos en una unidad de cuidados intensivos neonatales. Rev Investig Clínica. junio de 2005;57(3):425-33.

10. Sánchez Gómez, Diana Carolina; López Álvarez, Sara María. Factores relacionados a infecciones de sitio operatorio en pacientes hospitalizados - ProQuest [Internet]. Disponible en: https://search.proquest. com/openview/92ada291679bb7e0b10a428b8ee9881 4/1?pq-origsite=gscholar\&cbl=2037661

11. López Tagle D, Hernández Ferrer M, Saldivar Arias T, Sotolongo Hernández T, Valdés Dupeyrón O. Infección de la herida quirúrgica: Aspectos epidemiológicos. Rev Cuba Med Mil. junio de 2007;36(2):0-0.

12. Díaz-Agero-Pérez C, Pita-López MJ, Robustillo-Rodela A, Figuerola-Tejerina A, Monge-Jodrá V, others. Evaluación de la infección de herida quirúrgica en 14 hospitales de la Comunidad de Madrid: estudio de incidencia. Enfermedades Infecc Microbiol Clínica. 2011;29(4):257-62.

13. Pallares CJ, Martínez E. Mortality risk factors associated with healthcare infections in a tertiary level university hospital in Colombia. Biomédica. abril de 2014;34:148-55.

14. Vialat Soto V, Béquer M, José J, Hernández Alfonso $\mathrm{H}$, de la Rosa Rodríguez R. Infección de los sitios quirúrgicos: estudio de 1 año. Rev. Cuba Pediatría. marzo de 2008;80(1):0-0.

15. Carvajal R, Londoño Á. Factores de riesgo e infección del sitio quirúrgico en procedimientos de cirugía ortopédica con prótesis. Rev Chil Infectol. agosto de 2012;29(4):395-400.

16. Paranaguá TT de B, Bezerra ALQ, Moreira IA, Tobias GC, Silva AEB de C e. Indicadores de asistencia en un clínica quirúrgica. Enferm Glob. julio de 2016;15(43):228-39.

17. Domingues Duarte. Factores predisponentes de infección de la herida quirúrgica, revista de cirugía [Internet]. encolombia.com. 2014 [citado 15 de mayo de 2016]. Recuperado a partir de: https://encolombia. com/medicina/revistas-medicas/cirugia/vc-161/ cirugia16101-programa1/

18. Guanche Garcell H, Núñez Labrador L, Baxter Campaña M, Tolón Marín M, Morales Pérez C, Fresneda Septiem G, et al. Prevalencia de infección nosocomial en hospitales universitarios de La Habana, Cuba. An Med Interna. junio de 2006;23(6):269-71.

19. De La Rosa Muñoz K. Riesgo de infección en pacientes postquirúrgico relacionado con las medidas de bioseguridad y el cuidado de heridas. Hospital rafael serrano, la libertad 2013-2014. 28 de febrero de 2015 
[citado 24 de mayo de 2016]; Recuperado a partir de: http://repositorio.upse.edu.ec/handle/46000/1693.

20. López Tagle D, Hernández Ferrer M, Saldivar Arias T, Sotolongo Hernández T, Valdés Dupeyrón O. Infección de la herida quirúrgica: Aspectos epidemiológicos. Rev Cuba Med Mil. junio de 2007;36(2):0-0.

21. Chang F, Virgen N, Dalmau B, Mercedes $\mathrm{N}$ de las, Rodríguez M, Armando L, et al. Infección del sitio quirúrgico poscesárea. MEDISAN. mayo de 2016;20(5):596-603.

22. Infección de heridas quirúrgicas en cirugía general [Internet]. [citado 18 de agosto de 2017]. Disponible en: http://www.medigraphic.com/pdfs/circir/cc2011/cc114h.pdf.

23. Romero Sánchez RE, Landín Sorí M. Actualidad en infecciones quirúrgicas. Rev Arch Méd Camagüey. 2012;16(4):501-13.

24. Hernández Ruíz A, Llorente V, Arístides J, Fernández D, Iracema R, Cuello C, et al. Factores que influyen en la mortalidad en los pacientes graves con cirugía abdominal. Rev Cuba Cir. junio de 2014;53(2):134-44.

25. Ramos-Luces O, Molina N, Pillkahn W, Moreno J, Vieira A, Gómez J. Infección de heridas quirúrgicas en cirugía general. Rev Cir Cir. 2011;79(4):349-55.
26. Fernández López O, Rodríguez Fernández Z, Ochoa Maren G, Pineda Chacón J, García R, Ibrahim L. Factores de riesgo relacionados con las infecciones posoperatorias. MEDISAN. febrero de 2016;20(2):13242.

27. Bannura G, Guerra JF, Villarroel M, others. Infección de la herida operatoria en hernioplastía inguinal primaria. Rev. Chil Cir. 2006;58(5):330-5.

28. Molina RI, Bejarano M, García O. Infección del sitio operatorio en un hospital nivel II. Rev Colomb Cir. 2005;20(2):87-96.

29. Factores de riesgo relacionados con infección del sitio quirúrgico en cirugía electi va [Internet]. [citado 19 de agosto de 2017]. Disponible en: http://www.medigraphic.com/pdfs/circir/cc-2014/cc141g.pdf.

30. Hidalgo LF, Marroquín JE, Antigoni J, Samalvides F. Prevalencia de infecciones hospitalarias en un hospital peruano de nivel IV, en el año 2008. Rev Medica Hered. 2011;22(2):76-81. 